\title{
Need of Pharmacovigilance in AYUSH Drugs
}

\section{Rajanandh MG and Chamundeeswari $D^{*}$}

Faculty of Pharmacy, Sri Ramachandra University, Porur, Chennai-600116, India

*Corresponding author: Chamundeeswari D, Principal cum Professor, Faculty of Pharmacy, Sri Ramachandra University, Porur, Chennai-600116, India, Tel: 91-9444474076; E-mail: dvchamu@gmail.com

Received date: January 10, 2017; Accepted date: January 13, 2017; Published date: January 20, 2017

Copyright: (c) 2017 Chamundeeswari D. This is an open-access article distributed under the terms of the Creative Commons Attribution License, which permits unrestricted use, distribution, and reproduction in any medium, provided the original author and source are credited.

\section{Introduction}

Faculty of Pharmacy, Sri Ramachandra University in association with Society for Ethnopharmacology-Chennai chapter organized a National Seminar on "Pharmacovigilance of AYUSH drugs" on $19^{\text {th }}$ January 2016, at Sri Ramachandra University. A session on need of pharmacovigilance in AYUSH drugs was discussed and the salient points are summarized.

In India, Ayurveda, Siddha and Unani (ASU) systems of medicine are considered to be the oldest system of medicines. They prescribe drugs of herbal, mineral, metallic and animal origin for the treatment of many diseases. Being time tested systems of medicine and majority of the drugs used by them are of herbal origin these systems are considered to be safe. The use of ASU medicines continues to expand rapidly across the world. The interest and demand for ASU system of medicine is day-by-day increasing both within India and abroad.

Since people are more attractive towards ASU system and their awareness about ASU also increased dramatically, they are more concern about the safety of ASU system of practice. For the purpose of rationalization, there is a need for monitoring safety and efficacy of drugs of these medical systems and this is a matter of highest concern in current scenario. Though the traditional medical practices are deemed to be safest, few adverse effects were reported which are basically due to the non-judicial use, or use without the prescription, guidance or without supervision of a qualified medical practitioner etc. Hence the modern world demands a database for safe use of traditional medicine.

It is observed that few of these ASU drugs are being consumed, by patients, as OTC drug. They are sold as either herbal medicines or herbal products in different health-care settings. Due to inadequate regulatory measures, largely uncontrolled distribution channels either in form of mail order or internet sales and poor quality control systems, improper administration, some adverse events has also been reported.

\section{Present Status}

In India, till 2007, adverse drug reactions related to Indian systems of medicine were reported through National Pharmacovigilance programme. Considering the importance of drug safety, Department of AYUSH, Ministry of Health \& Family Welfare, Government launched the National Pharmacovigilance Programme for reporting the adverse drug reaction for Ayurveda, Siddha and Unani (ASU) drugs on 29.09.2008. A Protocol of National Pharmacovigilance Programme for ASU (NPP-ASU) drugs was published by AYUSH, in collaboration with WHO, in order to have a proper documentation, to regulate, monitor and control the activities of Pharmacovigilance. Three tier systems, viz. one National Pharmacovigilance Centre (NPC) and eight Regional Pharmacovigilance Centers (RPC) and thirty Peripheral Pharmacovigilance Centres (PPC) were set up throughout the country. Institute for Post Graduate Teaching \& Research in Ayurveda, Gujarat Ayurved University, Jamnagar, was designated as the National Pharmacovigilance Resource Centre (NPRC-ASU).

\section{ASU Drugs and their Risk Factors}

In Indian systems of medicines, drugs are of herbal, mineral, metallic or animal origin. As that of the conventional medicines, these drugs can also cause adverse drug events/reactions which are characteristic in nature to accompany the therapeutic efficacy of the drugs. During last seven years, under National Pharmacovigilance programme for ASU drugs, it is observed that, the majority of adverse events reported related to the use of ASU medicines, are attributable either to poor product quality or to improper use and can be categorized under two headings i.e. drug and clinics related.

\section{Drug related factors}

Quality of the ASU drugs may be hampered by poor quality raw material, plant preparations and not prepared following specific procedures. Good dispensing practices ensure that an effective form of the correct medicine is delivered to the right patient, in the correct dosage and quantity, with clear instructions, and in a package that maintains the potency of the medicine. It is observed that in many ASU hospitals drugs are dispensed in loose packets with an instruction to the patient to take the drug with an approximate weight basis. The efficacy of the drug also depends on the correct administration of it as mentioned in the form of prescribing information/package inserts or leaflets. It is observed that maximum ASU drugs available in the market do not contain required prescribed mandatory information, in the package insert. Further, in case of proprietary ASU formulations in addition to premarketing safety evaluation post marketing surveillance should also be given importance.

\section{Clinic related factors}

ASU systems of medicine have their own fundamental principles and have their own theory of diagnosis and treatment guidelines. Each drug is supposed to be administered with specific guidelines to the patient, depending upon the patient's prakruti, age, gender and the disease conditions etc. Pharmacopoeias of these systems of medicine have clear cut guidelines in this regard. A physician having concrete knowledge of theory and adequate practice experience of his/her own system of medicine should only prescribe these drugs. It has become common practice that ASU drugs are prescribed in combination with conventional medicines. Inappropriate combinations may however be another common cause of adverse drug reactions. 


\section{Future Prospective}

Pharmacovigilance practice is the need of hour for all systems of medicine including Indian Systems of Medicine, as it ensures patients safety, more scientific and up to date. Following are certain aspects where more emphasis is needed for better pharmacovigilance practice in ASU system of medicines.

- Strengthen education, training and publicity.

- Revitalization of an effective Pharmacovigilance system for ASU drugs

- Prioritize the focus of ASU drugs safety surveillance.

- Strengthen the roles of pharmaceutical manufacturers as the main body of ASU drugs post-marketing risk management.

- Promote the rational use of ASU drugs.

- Communicate safety information to the relevant agencies for cooperation to identify the nature of the ADRs

- Establish an international coordinating database for adverse reactions reporting and promote signal detection.

\section{Conclusion}

Pharmacovigilance practice is the need of hour for all systems of medicine including Indian Systems of Medicine, as it helps to prove this system safe, more scientific and up to date in modern terms. It is an absolute requirement to ensure public safety and to promote the healthy development of ASU systems of Medicine. All the stake holders of ASU systems of medicine need to be educated through intensive training and publicity regarding pharmacovigilance aspects of these drugs and Government and pharmaceutical sectors should take initiation in this regard by providing more financial assistance through budgetary provisions. The safety of ASU drugs is a concern throughout the whole life period of the drug. Quality control remains one of the main issues in ASU drugs safety concerns. Standardization and enforcement of GMP and manufacturing guidelines will support any safety initiative. Steps should be taken to strengthen ASU pharmaceutical manufacturers' responsibility and awareness of drug quality and of the value of basic research work on their drug products. Clinical use should be regulated by adopting Good Clinical Practice of ASU system to avoid the Adverse drug Reactions. Cooperation and sharing of information among the related drug regulating agencies should also be promoted.

\section{Acknowledgement}

The authors would like to thank the management of Sri Ramachandra University-Chennai, ICMR-New Delhi, AYUSH-New Delhi and Society for Ethanopharmacology-Chennai Chapter for their contribution towards the successful conduct of the seminar. 